\title{
An extremal problem for set families generated with the union and symmetric difference operations
}

\author{
Yuejian Peng*, Papa Sissokho and Cheng Zhao
}

\begin{abstract}
Let $\mathcal{G}$ be a family of sets and let $\cup^{n} \mathcal{G}$ be the family of sets obtained by taking all unions of $k$ sets of $\mathcal{G}$ with $1 \leq k \leq n$. We define the half-life of $\mathcal{G}$ with respect to the union operation, denoted by $h_{\cup}(\mathcal{G})$, to be the smallest integer $n$ such that some $x \in \cup_{A \in \mathcal{G}} A$ appears in at least half of the sets in $\cup^{n} \mathcal{G}$. If no such $n$ exists, then we define it as $\infty$. We also define the half-life of $\mathcal{G}$ with respect to the symmetric difference operation in a similar fashion and denote it by $h_{\Delta}(\mathcal{G})$. In this paper, we establish several bounds for $h_{\cup}(\mathcal{G})$ and $h_{\Delta}(\mathcal{G})$. As a byproduct, we confirm Fránkl's union-closed conjecture for some special cases.
\end{abstract}

AMS 2000 SUBJECT CLASSIFICATIONS: 05D05.

KEYWORDS AND PHRASES: Union-closed sets, union-closed conjecture, half-life.

\section{Introduction}

The symmetric difference of two sets $A$ and $B$ is $A \Delta B=(A \backslash B) \cup(B \backslash A)$. Let $\mathcal{G}$ be a family of sets and for any positive integer $n \leq|\mathcal{G}|$, define

$$
\cup^{n} \mathcal{G}=\left\{A_{i_{1}} \cup \cdots \cup A_{i_{k}}: \quad 1 \leq k \leq n \text { and } A_{i_{j}} \in \mathcal{G} \text { for } 1 \leq j \leq k\right\}
$$

and

$$
\Delta^{n} \mathcal{G}=\left\{A_{i_{1}} \Delta \cdots \Delta A_{i_{k}}: \quad 1 \leq k \leq n \text { and } A_{i_{j}} \in \mathcal{G} \text { for } 1 \leq j \leq k\right\} .
$$

Definition 1. A family of sets $\mathcal{F}$ is union-closed (or $\Delta$-closed) if it is closed under union (or symmetric difference), i.e, for any $A, B \in \mathcal{F}$, we have $A \cup B \in$ $\mathcal{F}$ (or $A \Delta B \in \mathcal{F})$.

Let $\mathcal{F}$ be a union-closed family of sets. We call $\mathcal{F}$ non-trivial if it contains a non-empty set. A generating set of $\mathcal{F}$ is a subfamily of sets $\mathcal{G} \subseteq \mathcal{F}$ such that

*Supported by the National Natural Science Foundation of China (No. 11271116). 
$\mathcal{F}$ is obtained by taking all the possible unions of sets in $\mathcal{G}$. Equivalently, we have

$$
\mathcal{F}=\cup^{m} \mathcal{G} \text {, where } m=|\mathcal{G}| \text {. }
$$

For any family of sets $\mathcal{F}$, the ground set of $\mathcal{F}$ is defined by $\mathcal{F}_{\text {gd }}=\cup_{A \in \mathcal{F}} A$. The union-closed conjecture, due to Fránkl [4], can be stated as follows.

Conjecture 2. For any non-trivial union-closed family of sets $\mathcal{F}$, there exists an element $x \in \mathcal{F}_{\mathrm{gd}}$ which appears in at least half of the sets of $\mathcal{F}$.

This simply stated conjecture turned out to be quite difficult to solve. Partial results that support Conjecture 2 can be found in [1, 3-5, 7-12] and the references therein.

If one takes a $\Delta$-closed family of sets, then it is easy to show that the conclusion of Conjecture 2 holds. More precisely, the following proposition holds.

Proposition 3. Let $\mathcal{F}$ be any non-trivial $\Delta$-closed family of sets with ground set $\mathcal{F}_{\mathrm{gd}}$. Then, any $x \in \mathcal{F}_{\mathrm{gd}}$ appears in at least half of the sets in $\mathcal{F}$.

Proof. Let $\mathcal{F}=\left\{A_{1}, \ldots, A_{l}\right\}$ be a non-trivial $\Delta$-closed family of sets on the ground set $\mathcal{F}_{g}$. For any $x \in \mathcal{F}_{g}$, there exists at least one $A_{i}$ containing $x$. Suppose that the number of sets in $\mathcal{F}$ containing $x$ is less than $\frac{l}{2}$. Without loss of generality, assume that $x \in A_{l}$ and each $A_{1}, \ldots, A_{\left\lceil\frac{l+1}{2}\right\rceil}$ does not contain $x$. Then the sets $A_{l} \Delta A_{i}$, where $1 \leq i \leq\left\lceil\frac{l+1}{2}\right\rceil$, are pairwise distinct and each of them contains $x$. Since $\mathcal{F}$ is union-closed, all these sets are in $\mathcal{F}$, and this contradicts the assumption that the number of sets in $\mathcal{F}$ containing $x$ is less than $\frac{l}{2}$.

Let $\mathcal{G}$ be a non-empty family of $l \geq 2$ sets and let $n$ be a nonnegative integer. We define the half-life of $\mathcal{G}$ with respect to $\cup$ to be the smallest integer $n$ such that some $x \in \mathcal{G}_{\text {gd }}$ appears in at least half of the sets in $\cup^{n} \mathcal{G}$. If no such $n$ exists, then we define it as $\infty$. We use $h_{\cup}(\mathcal{G})$ to denote the half-life of $\mathcal{G}$ with respect to $\cup$. The half-life of $\mathcal{G}$ with respect to $\Delta$ is defined similarly and denoted by $h_{\Delta}(\mathcal{G})$. The following problem will help us to understand the union-closed conjecture.

Problem 4. Determine $h_{\cup}(\mathcal{G})$ and $h_{\Delta}(\mathcal{G})$.

This rest of the paper is organized as follows. We give some bounds for $h_{\Delta}(\mathcal{G})$ and $h_{\cup}(\mathcal{G})$ in Sections 2 and 3 respectively. In Section 4, we confirm the union-closed conjecture for some special cases. 


\section{Some bounds on $h_{\Delta}(\mathcal{G})$}

Let $\mathcal{G}$ be a family with $l \geq 2$ sets and $x \in \mathcal{G}_{\text {gd }}$. For any positive integer $n$, let $\Delta^{n}(\mathcal{G}, x)$ be the family of sets in $\Delta^{n} \mathcal{G}$ containing $x$. Let $\Delta^{n}(\mathcal{G}, \bar{x})$ be the family of sets in $\Delta^{n} \mathcal{G}$ not containing $x$. We define the half-life of $x \in \mathcal{G}_{\text {gd }}$ with respect to $\Delta$, denoted by $h_{\Delta}(\mathcal{G}, x)$, to be the minimum between $\infty$ and the smallest integer $n$ such that

$$
\frac{\left|\Delta^{n}(\mathcal{G}, x)\right|}{\left|\Delta^{n}(\mathcal{G})\right|} \geq \frac{1}{2}
$$

Thus,

$$
h_{\Delta}(\mathcal{G})=\min _{x \in \mathcal{G}_{\text {gd }}} h_{\Delta}(\mathcal{G}, x) .
$$

If $l=2$, then $h_{\Delta}(\mathcal{G}, x)=1$ for any $x \in \mathcal{G}_{\text {gd }}$. So we assume $l \geq 3$.

Proposition 5. For any family $\mathcal{G}$ with $l \geq 3$ sets and for any $x \in \mathcal{G}_{\text {gd }}$, $h_{\Delta}(\mathcal{G}, x) \leq|\mathcal{G}|$ holds.

Proof. Since $\Delta^{l} \mathcal{G}$ is $\Delta$-closed for $l=|\mathcal{G}|$, Proposition 3 implies that

$$
h_{\Delta}(\mathcal{G}, x) \leq|\mathcal{G}|
$$

In order to state our main result in this section, we need the following definition.

Definition 6. A family of non-empty sets $\mathcal{S}=\left\{A_{1}, \ldots, A_{l}\right\}$ is called linearly independent if for any integer $j$ with $1 \leq j \leq l$ and for all indices $i_{1}, \ldots, i_{s} \in$ $\{1, \ldots, l\} \backslash\{j\}$, we have $A_{j} \neq A_{i_{1}} \Delta \cdots \Delta A_{i_{s}}$.

Lemma 7 will be used in Theorems 8 and 9 .

Lemma 7. Let $\mathcal{S}=\left\{A_{1}, \ldots, A_{l}\right\}$ be a linearly independent family of sets. Let $1 \leq i_{1}<\cdots<i_{s} \leq l$ and $1 \leq j_{1}<\cdots<j_{t} \leq l$. Then, $A_{i_{1}} \Delta \cdots \Delta A_{i_{s}}=$ $A_{j_{1}} \Delta \cdots \Delta A_{j_{t}}$ if and only if $\left\{i_{1}, \ldots, i_{s}\right\}=\left\{j_{1}, \ldots, j_{t}\right\}$.

Proof. Let $I=\left\{i_{1}, \ldots, i_{s}\right\}$ and $J=\left\{j_{1}, \ldots, j_{t}\right\}$. Suppose that $I \neq J$ and

$$
A_{i_{1}} \Delta \cdots \Delta A_{i_{s}}=A_{j_{1}} \Delta \cdots \Delta A_{j_{t}}
$$

We may assume that $I \cap J=\emptyset$. Indeed, for each $i \in I \cap J$ we can operate by $A_{i} \Delta$ on both sides of (3) and consider resulting sets $I^{\prime}=I \backslash I \cap J$ and 
$J^{\prime}=J \backslash I \cap J$. With the assumption $I \cap J=\emptyset$, we can now operate by $A_{i_{1}} \Delta \cdots \Delta A_{i_{s-1}} \Delta$ on both sides of (3) to obtain

$$
A_{i_{s}}=A_{i_{1}} \Delta \cdots \Delta A_{i_{s-1}} \Delta A_{j_{1}} \Delta \cdots \Delta A_{j_{t}}
$$

This contradicts the linear independence of $\mathcal{S}$.

Theorem 8. Let $\mathcal{S}=\left\{A_{1}, \ldots, A_{l}\right\}$ be a linearly independent family sets with $l \geq 3$. For $x \in \cup_{i=1}^{l} A_{i}$, let $q_{x}$ be the number of sets in $\mathcal{S}$ containing $x$.

(a) If $q_{x}$ is even, then $h_{\Delta}(\mathcal{S}, x) \leq\left\lceil\frac{l-1}{2}\right\rceil$.

(b) If $q_{x}$ is odd and $h_{\Delta}(\mathcal{S}, x) \neq l$, then $h_{\Delta}(\mathcal{S}, x) \leq\left\lfloor\frac{l-1}{2}\right\rfloor$.

Proof. Since $\Delta^{l} \mathcal{S}$ is $\Delta$-closed, Proposition 3 yields

$$
\left|\Delta^{l}(\mathcal{S}, x)\right| \geq\left|\Delta^{l}(\mathcal{S}, \bar{x})\right|
$$

We simply write $q_{x}$ as $q$ throughout the proof. Without loss of generality, we assume that $x \in A_{i}$ for $1 \leq i \leq q$ and $x \notin A_{i}$ for $i>q$. Note that a set in $\Delta^{n}(\mathcal{S}, x)$ must be of the form $A_{i_{1}} \Delta \cdots \Delta A_{i_{s}}$, where there are an odd number of indices $i_{j} \in\left\{i_{1}, \ldots, i_{s}\right\}$ such that $i_{j} \leq q$. Similarly, a set in $\Delta^{n}(\mathcal{S}, \bar{x})$ must be $\emptyset$ or of the form $A_{i_{1}} \Delta \cdots \Delta A_{i_{s}}$, where there are an even number of indices $i_{j} \in\left\{i_{1}, \ldots, i_{s}\right\}$ such that $i_{j} \leq q$.

(a) $q$ is even. For any positive integer $n \leq l-2$, define a function $f$ on $\Delta^{n}(\mathcal{S}, x)$ by

$$
f\left(A_{i_{1}} \Delta \cdots \Delta A_{i_{s}}\right)=A_{j_{1}} \Delta \cdots \Delta A_{j_{l-s}}
$$

where $\left\{j_{1}, \ldots, j_{l-s}\right\}=\{1, \ldots, l\} \backslash\left\{i_{1}, \ldots, i_{s}\right\}$. By Lemma $7, f$ is a oneto-one function. Since $q$ is even, $f$ is an onto function from $\Delta^{n}(\mathcal{S}, x)$ to $\Delta^{l}(\mathcal{S}, x)-\Delta^{l-n-1}(\mathcal{S}, x)$, which is the set

$$
\left\{A_{i_{1}} \Delta \cdots \Delta A_{i_{t}}: x \in A_{i_{1}} \Delta \cdots \Delta A_{i_{t}}, i_{1}<\cdots<i_{t}, l-n \leq t \leq l\right\} .
$$

Therefore,

$$
\left|\Delta^{n}(\mathcal{S}, x)\right|=\left|\Delta^{l}(\mathcal{S}, x)\right|-\left|\Delta^{l-n-1}(\mathcal{S}, x)\right|
$$

Similarly, define a function $g$ on $\Delta^{n}(\mathcal{S}, \bar{x})$ by

$$
g\left(A_{i_{1}} \Delta \cdots \Delta A_{i_{s}}\right)=A_{j_{1}} \Delta \cdots \Delta A_{j_{l-s}}
$$

where $\left\{j_{1}, \ldots, j_{l-s}\right\}=\{1, \ldots, l\} \backslash\left\{i_{1}, \ldots, i_{s}\right\}$, and

$$
g(\emptyset)=A_{1} \Delta \cdots \Delta A_{l} \text { if } n \geq 2 .
$$


Then $g$ is a one-to-one and onto function from $\Delta^{n}(\mathcal{S}, \bar{x})$ to $\Delta^{l}(\mathcal{S}, \bar{x})-$ $\Delta^{l-n-1}(\mathcal{S}, \bar{x})$. Therefore,

$$
\left|\Delta^{n}(\mathcal{S}, \bar{x})\right|=\left|\Delta^{l}(\mathcal{S}, \bar{x})\right|-\left|\Delta^{l-n-1}(\mathcal{S}, \bar{x})\right| .
$$

By (4), (5) and (6),

$$
\left|\Delta^{n}(\mathcal{S}, x)\right|+\left|\Delta^{l-n-1}(\mathcal{S}, x)\right| \geq\left|\Delta^{n}(\mathcal{S}, \bar{x})\right|+\left|\Delta^{l-n-1}(\mathcal{S}, \bar{x})\right| .
$$

Thus, $\left|\Delta^{n}(\mathcal{S}, x)\right| \geq\left|\Delta^{n}(\mathcal{S}, \bar{x})\right|$ or $\left|\Delta^{l-n-1}(\mathcal{S}, x)\right| \geq \mid \Delta^{l-n-1}(\mathcal{S}, \bar{x} \mid$. If we take $n=\left\lfloor\frac{l-1}{2}\right\rfloor$, then we get

$$
h_{\Delta}(\mathcal{S}, x) \leq\left\lfloor\frac{l-1}{2}\right\rfloor \text { or } h_{\Delta}(\mathcal{S}, x) \leq\left\lceil\frac{l-1}{2}\right\rceil .
$$

(b) $q$ is odd. For any positive integer $n \leq l-1$, define a function $f$ on $\Delta^{n}(\mathcal{S}, x)$ by

$$
f\left(A_{i_{1}} \Delta \cdots \Delta A_{i_{s}}\right)=A_{j_{1}} \Delta \cdots \Delta A_{j_{l-s}}
$$

where $\left\{j_{1}, \ldots, j_{l-s}\right\}=\{1, \ldots, l\} \backslash\left\{i_{1}, \ldots, i_{s}\right\}$.

By Lemma $7, f$ is a one-to-one function. Since $q$ is odd, $f$ is an onto function from $\Delta^{n}(\mathcal{S}, x)$ to $\Delta^{l}(\mathcal{S}, \bar{x})-\Delta^{l-n-1}(\mathcal{S}, \bar{x})$, where $\Delta^{0}(\mathcal{S}, \bar{x})$ is the family containing only the empty set $\emptyset$. Therefore,

$$
\left|\Delta^{n}(\mathcal{S}, x)\right|=\left|\Delta^{l}(\mathcal{S}, \bar{x})\right|-\left|\Delta^{l-n-1}(\mathcal{S}, \bar{x})\right| .
$$

Similarly, define a function $g$ on $\Delta^{n}(\mathcal{S}, \bar{x})$ by

$$
g\left(A_{i_{1}} \Delta \cdots \Delta A_{i_{s}}\right)=A_{j_{1}} \Delta \cdots \Delta A_{j_{l-s}}
$$

where $\left\{j_{1}, \ldots, j_{l-s}\right\}=\{1, \ldots, l\} \backslash\left\{i_{1}, \ldots, i_{s}\right\}$, and

$$
g(\emptyset)=A_{1} \Delta \cdots \Delta A_{l} \text { if } n \geq 2 .
$$

Then $g$ is a one-to-one and onto function from $\Delta^{n}(\mathcal{S}, \bar{x})$ to $\Delta^{l}(\mathcal{S}, x)-$ $\Delta^{l-n-1}(\mathcal{S}, x)$, where $\Delta^{0}(\mathcal{S}, x)$ is the empty family. Therefore,

$$
\left|\Delta^{n}(\mathcal{S}, \bar{x})\right|=\left|\Delta^{l}(\mathcal{S}, x)\right|-\left|\Delta^{l-n-1}(\mathcal{S}, x)\right| .
$$

By (4), (7) and (8),

$$
\left|\Delta^{n}(\mathcal{S}, x)\right|-\left|\Delta^{n}(\mathcal{S}, \bar{x})\right| \leq\left|\Delta^{l-n-1}(\mathcal{S}, x)\right|-\left|\Delta^{l-n-1}(\mathcal{S}, \bar{x})\right| .
$$


Swapping $n$ with $l-n-1$ yields

$$
\left|\Delta^{n}(\mathcal{S}, x)\right|-\left|\Delta^{n}(\mathcal{S}, \bar{x})\right| \geq\left|\Delta^{l-n-1}(\mathcal{S}, x)\right|-\left|\Delta^{l-n-1}(\mathcal{S}, \bar{x})\right| .
$$

Thus,

$$
\left|\Delta^{n}(\mathcal{S}, x)\right|-\left|\Delta^{n}(\mathcal{S}, \bar{x})\right|=\left|\Delta^{l-n-1}(\mathcal{S}, x)\right|-\left|\Delta^{l-n-1}(\mathcal{S}, \bar{x})\right| .
$$

So if $h_{\Delta}(\mathcal{S}, x) \neq l$, then there exists at least one $n \leq l-1$ such that

$$
h_{\Delta}(\mathcal{S}, x) \leq \min \{n, l-n-1\} \leq\left\lfloor\frac{l-1}{2}\right\rfloor .
$$

Theorem 9. Let $\mathcal{G}=\left\{A_{1}, \ldots, A_{l}\right\}$ be a linearly independent family of $l \geq 3$ sets. Let $q_{x}$ be the number of sets in $\mathcal{G}$ containing $x$.

(a) $h_{\Delta}(\mathcal{G}, x)=2$ if and only if $\frac{(l+1)-\sqrt{l-1}}{2} \leq q_{x}<\frac{l}{2}$.

(b) If $q_{x}=1$, then $h_{\Delta}(\mathcal{G}, x)=l$.

(c) If $q_{x}=2$ and $l \geq 5$, then $h_{\Delta}(\mathcal{G}, x)=\left\lceil\frac{l-1}{2}\right\rceil$.

(d) If $q_{x}=3$ and $l \geq 7$, then $h_{\Delta}(\mathcal{G}, x)=\left\lceil\frac{l-1-\sqrt{l-1}}{2}\right\rceil$.

Proof. We simply write $q_{x}$ as $q$ throughout the proof. Without loss of generality, we assume that $x \in A_{i}$ for $1 \leq i \leq q$ and $x \notin A_{i}$ for $i>q$.

Note that a set in $\Delta^{n}(\mathcal{G}, x)$ must be of the form $A_{i_{1}} \Delta \cdots \Delta A_{i_{s}}$, where there are an odd number of indices $i_{j} \in\left\{i_{1}, \ldots, i_{s}\right\}$ such that $i_{j} \leq q$. Similarly, a set in $\Delta^{n}(\mathcal{G}, \bar{x})$ must be $\emptyset$ or of the form $A_{i_{1}} \Delta \cdots \Delta A_{i_{s}}$, where there are an even number of indices $i_{j} \in\left\{i_{1}, \ldots, i_{s}\right\}$ such that $i_{j} \leq q$. Also recall that, by Lemma 7 , all sets $A_{i_{1}} \Delta \cdots \Delta A_{i_{s}}$ with $i_{1}<\cdots<i_{s}$ and $1 \leq s \leq l$ are pairwise distinct. So for $n \geq 2$, we obtain

(9) $\left|\Delta^{n}(\mathcal{G}, x)\right|=\sum_{k=0}^{\min \left\{\left\lfloor\frac{q-1}{2}\right\rfloor,\left\lfloor\frac{n-1}{2}\right\rfloor\right\}}\left(\begin{array}{c}q \\ 2 k+1\end{array}\right) \sum_{j=0}^{\min \{l-q, n-(2 k+1)\}}\left(\begin{array}{c}l-q \\ j\end{array}\right)$,

and

$$
\left|\Delta^{n}(\mathcal{G}, \bar{x})\right|=\sum_{k=0}^{\min \left\{\left\lfloor\frac{q}{2}\right\rfloor,\left\lfloor\frac{n}{2}\right\rfloor\right\}}\left(\begin{array}{c}
q \\
2 k
\end{array}\right) \sum_{j=0}^{\min \{l-q, n-2 k\}}\left(\begin{array}{c}
l-q \\
j
\end{array}\right) .
$$

To show that $\left|\Delta^{n}(\mathcal{G}, x)\right| \geq \frac{1}{2}\left|\Delta^{n} \mathcal{G}\right|$, it is equivalent to show that $\left|\Delta^{n}(\mathcal{G}, x)\right|-$ $\left|\Delta^{n}(\mathcal{G}, \bar{x})\right| \geq 0$. 
(a) Now assume $h_{\Delta}(\mathcal{G}, x)=2$. Since

$$
\left|\Delta^{2} \mathcal{G}\right|=1+\left(\begin{array}{l}
l \\
1
\end{array}\right)+\left(\begin{array}{l}
l \\
2
\end{array}\right) \text { and }\left|\Delta^{2}(\mathcal{G}, x)\right|=q+q(l-q),
$$

then $\left|\Delta^{2}(\mathcal{G}, x)\right| \geq \frac{1}{2}\left|\Delta^{2} \mathcal{G}\right|$ if and only if $q+q(l-q) \geq \frac{1}{2}\left(1+l+\left(\begin{array}{l}l \\ 2\end{array}\right)\right)$.

Solving the previous inequality for $q$ yields $\frac{(l+1)-\sqrt{l-1}}{2} \leq q \leq \frac{(l+1)+\sqrt{l-1}}{2}$. Since $h_{\Delta}(\mathcal{G}, x)=1$ when $q \geq \frac{l}{2}$, we conclude that $\frac{(l+1)-\sqrt{l-1}}{2} \leq q<\frac{l}{2}$.

(b) If $q=1$, then for $2 \leq n \leq l-1$,

$$
\begin{aligned}
\left|\Delta^{n}(\mathcal{G}, x)\right|-\left|\Delta^{n}(\mathcal{G}, \bar{x})\right| & =\sum_{j=0}^{n-1}\left(\begin{array}{c}
l-1 \\
j
\end{array}\right)-\sum_{j=0}^{n}\left(\begin{array}{c}
l-1 \\
j
\end{array}\right) \\
& =-\left(\begin{array}{c}
l-1 \\
n
\end{array}\right) \\
& <0 .
\end{aligned}
$$

By Proposition $5, h_{\Delta}(\mathcal{G}, x)=l$.

(c) If $q=2$ and $l \geq 5$, then for $2 \leq n \leq l-2$,

$$
\begin{aligned}
\left|\Delta^{n}(\mathcal{G}, x)\right|-\left|\Delta^{n}(\mathcal{G}, \bar{x})\right| & =2 \sum_{j=0}^{n-1}\left(\begin{array}{c}
l-2 \\
j
\end{array}\right)-\left(\sum_{j=0}^{n}\left(\begin{array}{c}
l-2 \\
j
\end{array}\right)+\sum_{j=0}^{n-2}\left(\begin{array}{c}
l-2 \\
j
\end{array}\right)\right) \\
& =\left(\begin{array}{c}
l-2 \\
n-1
\end{array}\right)-\left(\begin{array}{c}
l-2 \\
n
\end{array}\right) \\
& \geq 0
\end{aligned}
$$

where the last inequality holds if and only if $n \geq\left\lceil\frac{l-1}{2}\right\rceil$. So $h_{\Delta}(\mathcal{G}, x)=\left\lceil\frac{l-1}{2}\right\rceil$.

(d) If $q=3$ and $l \geq 7$, then for $2 \leq n \leq l-3$,

$$
\begin{aligned}
& \left|\Delta^{n}(\mathcal{G}, x)\right|-\left|\Delta^{n}(\mathcal{G}, \bar{x})\right|=3 \sum_{j=0}^{n-1}\left(\begin{array}{c}
l-3 \\
j
\end{array}\right)+\sum_{j=0}^{n-3}\left(\begin{array}{c}
l-3 \\
j
\end{array}\right) \\
& -\left(\sum_{j=0}^{n}\left(\begin{array}{c}
l-3 \\
j
\end{array}\right)+3 \sum_{j=0}^{n-2}\left(\begin{array}{c}
l-3 \\
j
\end{array}\right)\right) \\
& =2\left(\begin{array}{c}
l-3 \\
n-1
\end{array}\right)-\left(\begin{array}{c}
l-3 \\
n
\end{array}\right)-\left(\begin{array}{c}
l-3 \\
n-2
\end{array}\right) \\
& \geq 0 \text {, }
\end{aligned}
$$


where the last inequality holds if and only if $\left\lceil\frac{l-1-\sqrt{l-1}}{2}\right\rceil \leq n \leq\left\lceil\frac{l-1+\sqrt{l-1}}{2}\right\rceil$. So $h_{\Delta}(\mathcal{G}, x)=\left\lceil\frac{l-1-\sqrt{l-1}}{2}\right\rceil$.

Remark 10. It follows from the proof of Theorem 9 part (d) that $\frac{\left|\Delta^{n}(\mathcal{G}, x)\right|}{\left|\Delta^{n} \mathcal{G}\right|}$ is not necessarily monotone with respect to $n$.

Theorem 11. Let $\mathcal{G}=\left\{A_{1}, \ldots, A_{m}\right\}$ be a family of sets and let $l \geq 3$ be the maximum size of a linearly independent subset $\mathcal{S}$ of $\mathcal{G}$. For $x \in \cup_{i=1}^{l} A_{i}$, let $q_{x}$ be the number of sets in $\mathcal{G}$ containing $x$.

(a) If $q_{x}$ is even, then $h_{\Delta}(\mathcal{G}, x) \leq l-1$.

(b) If $q_{x}$ is odd and $\mathcal{S} \subset \mathcal{G}$, then $h_{\Delta}(\mathcal{G}, x) \leq l-1$.

Proof. Let $\mathcal{S}=\left\{A_{1}, \ldots, A_{l}\right\}$ be a maximum linearly independent subset of $\mathcal{G}$. Then for every non-empty set $A_{j} \in \mathcal{G}$, there exists $1 \leq i_{1}<\cdots<i_{s} \leq l$ such that $A_{j}=A_{i_{1}} \Delta \cdots \Delta A_{i_{s}}$. Since

$$
\Delta^{l} \mathcal{G}=\Delta^{l} \mathcal{S}=\{\emptyset\} \cup\left\{A_{i_{1}} \Delta \cdots \Delta A_{i_{s}}: 1 \leq i_{1}<\cdots<i_{s} \leq l\right\},
$$

both $\Delta^{l} \mathcal{G}$ and $\Delta^{l} \mathcal{S}$ are $\Delta$-closed. Furthermore, $\Delta^{l-1} \mathcal{S}=\Delta^{l} \mathcal{S} \backslash\left\{A_{1} \Delta A_{3} \ldots\right.$ $\left.\Delta A_{l}\right\}$ and $\Delta^{l-1} \mathcal{G}$ is either $\Delta^{l-1} \mathcal{S}$ or $\Delta^{l} \mathcal{S}$.

(a) Assume that $q_{x}$ is even. If $\Delta^{l-1} \mathcal{G}=\Delta^{l} \mathcal{S}$, then $\Delta^{l-1} \mathcal{G}$ is $\Delta$-closed, and it follows from Proposition 3 that $h_{\Delta}(\mathcal{G}, x) \leq l-1$. So we may assume that $\Delta^{l-1} \mathcal{G}=\Delta^{l-1} \mathcal{S}$.

Our goal now is to show that $\left|\Delta^{l-1}(\mathcal{G}, x)\right| \geq\left|\Delta^{l-1} \mathcal{G}\right| / 2$. Since $\Delta^{l-1}(\mathcal{S}, x)=$ $\Delta^{l-1}(\mathcal{G}, x)$, it suffices to show that

$$
\left|\Delta^{l-1}(\mathcal{S}, x)\right| \geq\left|\Delta^{l-1} \mathcal{S}\right| / 2 .
$$

By Proposition $3,\left|\Delta^{l}(\mathcal{S}, x)\right| \geq\left|\Delta^{l} \mathcal{S}\right| / 2$. Note that $\left|\Delta^{l} \mathcal{S}\right|-\left|\Delta^{l-1} \mathcal{S}\right|=1$ since the only element in $\Delta^{l} \mathcal{S} \backslash \Delta^{l-1} \mathcal{S}$ is $A_{1} \Delta \cdots \Delta A_{l}$.

Since $q_{x}$ is even, then $A_{1} \Delta \cdots \Delta A_{l}$ does not contain $x$, and consequently $\left|\Delta^{l-1}(\mathcal{S}, x)\right|=\left|\Delta^{l}(\mathcal{S}, x)\right|$. Hence, $\left|\Delta^{l-1}(\mathcal{S}, x)\right| \geq\left|\Delta^{l-1} \mathcal{S}\right| / 2$.

(b) Assume that $q_{x}$ is odd and $\mathcal{S} \subseteq \mathcal{G}$. We claim that $\Delta^{l-1} \mathcal{G}=\Delta^{l} \mathcal{S}$. Note that $\Delta^{l-1} \mathcal{S} \subseteq \Delta^{l-1} \mathcal{G} \subseteq \Delta^{l} \mathcal{S}$ and $\Delta^{l} \mathcal{S} \backslash \Delta^{l-1} \mathcal{S}=\left\{A_{1} \Delta \cdots \Delta A_{l}\right\}$. Since $\mathcal{S} \subset \mathcal{G}$, then there exists $A_{i} \in \mathcal{G} \backslash \mathcal{S}$ such that $A_{i}$ is the symmetric difference of at least two sets in $\mathcal{S}$; therefore, $A_{1} \Delta \cdots \Delta A_{l} \in \Delta^{l-1} \mathcal{G}$ and consequently $\Delta^{l-1} \mathcal{G}=\Delta^{l} \mathcal{S}$. Then again it follows from Proposition 3 that $h_{\Delta}(\mathcal{G}, x) \leq l-1$ since $\Delta^{l-1} \mathcal{G}$ is $\Delta$-closed. 


\section{Some bounds on $h_{\cup}(\mathcal{G})$}

Let $\mathcal{G}$ be a family with $l \geq 2$ sets and $x \in \mathcal{G}_{\text {gd }}$. For any positive integer $n$, let $\cup^{n}(\mathcal{G}, x)$ be the family of sets in $\cup^{n} \mathcal{G}$ containing $x$. Let $\cup^{n}(\mathcal{G}, \bar{x})$ be the family of sets in $\cup^{n} \mathcal{G}$ not containing $x$. Recall that the half-life of $x \in \mathcal{G}_{\text {gd }}$ with respect to $\cup$, denoted by $h_{\cup}(\mathcal{G}, x)$, is the minimum between $\infty$ and the smallest integer $n$ such that

$$
\frac{\left|\cup^{n}(\mathcal{G}, x)\right|}{\left|\cup^{n}(\mathcal{G})\right|} \geq \frac{1}{2} .
$$

Thus,

$$
h_{\cup}(\mathcal{G})=\min _{x \in \mathcal{G}_{\text {gd }}} h_{\cup}(\mathcal{G}, x) .
$$

If the union-closed conjecture (Conjecture 2) is true, then $h_{\cup}(\mathcal{G}) \leq|\mathcal{G}|$. We know from Remark 10 that $\left|\Delta^{n}(\mathcal{G}, x)\right| /\left|\Delta^{n} \mathcal{G}\right|$ is not necessarily monotone with respect to $n$. It is interesting to investigate whether or not $\mid \cup^{n}$ $(\mathcal{G}, x)|/| \cup^{n} \mathcal{G} \mid$ is monotone with respect to $n$. If $\left|\cup^{n}(\mathcal{G}, x)\right| /\left|\cup^{n} \mathcal{G}\right|$ were monotone, then union-closed conjecture would hold if and only if $h_{\cup}(\mathcal{G}) \leq|\mathcal{G}|$.

We now provide two results about $h_{\cup}(\mathcal{G}, x)$ when its value is significantly less than $|\mathcal{G}|$.

Proposition 12. Let $\mathcal{G}=\cup_{i=1}^{l} \mathcal{G}_{i}$ be a family of sets, where $\mathcal{G}_{i}$ is a union closed family of sets and let $x$ be an element in the ground set of $\mathcal{G}$. Assume that $\left|\mathcal{G}_{i}\right|=g>1$ for $1 \leq i \leq l$ and $A_{i_{1}} \cup \cdots \cup A_{i_{s}} \neq A_{j_{1}} \cup \cdots \cup A_{j_{k}}$ for $\left\{i_{1}, \ldots, i_{s}\right\} \neq\left\{j_{1}, \ldots, j_{k}\right\}$, where $A_{i_{p}} \in \mathcal{G}_{i_{p}}$ for $1 \leq p \leq l$. Finally, assume that there is an $i_{0}$, with $1 \leq i_{0} \leq l$, such that $x$ belongs to all sets in $\mathcal{G}_{i_{0}}$ and $x$ does not belong to any other set. Then $h_{\cup}(\mathcal{G}, x) \leq l-1$ whenever $l \geq 1+\ln 2 / \ln (1+1 / g)$. Moreover, the union-closed conjecture is true for $\cup^{l} \mathcal{G}$, and thus $h_{\cup}(\mathcal{G}, x) \leq l=|\mathcal{G}| / g$ holds in general.

Proof. Since all $A_{i_{1}} \cup \cdots \cup A_{i_{s}}$ are pairwise distinct where $i_{1}<\cdots<i_{s}$ and each $A_{i_{j}}$ is from $\mathcal{G}_{j}, 1 \leq j \leq s$ and $1 \leq s \leq l$, then

$$
\left|\cup^{n} \mathcal{G}\right|=\sum_{j=1}^{n} g^{j}\left(\begin{array}{l}
l \\
j
\end{array}\right) .
$$

Since a set in $\cup^{n} \mathcal{G}$ containing $x$ must be of the form

$$
A_{i_{0}} \text { or } A_{i_{0}} \cup A_{i_{1}} \cup \cdots \cup A_{i_{s}}
$$


then

$$
\left|\cup^{n}(\mathcal{G}, x)\right|=\sum_{j=0}^{n-1} g \cdot g^{j}\left(\begin{array}{c}
l-1 \\
j
\end{array}\right)
$$

So,

$$
\begin{aligned}
2\left|\cup^{n}(\mathcal{G}, x)\right|= & +\sum_{j=1}^{n-1}\left(g^{j}\left(\begin{array}{c}
l-1 \\
j-1
\end{array}\right)+g^{j}\left(\begin{array}{c}
l-1 \\
j
\end{array}\right)\right) \\
& +\sum_{j=1}^{n-1}\left(g^{j+1}-g^{j}\right)\left(\begin{array}{c}
l-1 \\
j
\end{array}\right)+g^{n-1+1}\left(\begin{array}{c}
l-1 \\
n-1
\end{array}\right) \\
\geq & g+\sum_{j=1}^{n-1} g^{j}\left(\begin{array}{l}
l \\
j
\end{array}\right)+\sum_{j=1}^{n-1} g^{j}\left(\begin{array}{c}
l-1 \\
j
\end{array}\right)+g^{n-1+1}\left(\begin{array}{c}
l-1 \\
n-1
\end{array}\right) \\
= & \left|\cup^{n} \mathcal{G}\right|+g+\sum_{j=1}^{n-1} g^{j}\left(\begin{array}{c}
l-1 \\
j
\end{array}\right)+g^{n-1+1}\left(\begin{array}{c}
l-1 \\
n-1
\end{array}\right)-g^{n}\left(\begin{array}{l}
l \\
n
\end{array}\right),
\end{aligned}
$$

here we used the assumption $g>1$.

Case 1. If $n=l-1$, then

$$
\begin{aligned}
2\left|\cup^{l-1}(\mathcal{G}, x)\right| & \geq\left|\cup^{l-1} \mathcal{G}\right|+g+\sum_{j=1}^{l-2} g^{j}\left(\begin{array}{c}
l-1 \\
j
\end{array}\right)+g^{l-1}(l-1)-g^{l-1} l \\
& \geq\left|\cup^{l-1} \mathcal{G}\right|+\sum_{j=0}^{l-1} g^{j}\left(\begin{array}{c}
l-1 \\
j
\end{array}\right)-g^{l-1}+g^{l-1}(l-1)-g^{l-1} l \\
& =\left|\cup^{l-1} \mathcal{G}\right|+(1+g)^{l-1}-g^{l-1}+g^{l-1}(l-1)-g^{l-1} l \\
& =\left|\cup^{l-1} \mathcal{G}\right|+(1+g)^{l-1}-2 g^{l-1} .
\end{aligned}
$$

Therefore, $2\left|\cup^{l-1}(\mathcal{G}, x)\right| \geq\left|\cup^{l-1} \mathcal{G}\right|$ when $l \geq 1+\ln 2 / \ln (1+1 / g)$.

Case 2. If $n=l$, then

$$
2\left|\cup^{l}(\mathcal{G}, x)\right| \geq\left|\cup^{l} \mathcal{G}\right|+g+g^{l}\left(\begin{array}{l}
l-1 \\
l-1
\end{array}\right)-g^{l}\left(\begin{array}{l}
l \\
l
\end{array}\right)>\left|\cup^{l} \mathcal{G}\right|
$$

Therefore, we have shown that $h_{\cup}(\mathcal{G}) \leq l-1$ when $l>1+\ln 2 / \ln (1+1 / g)$. Moreover, the union-closed conjecture is true for $\cup^{l} \mathcal{G}$ and thus, $h_{\cup}(\mathcal{G}) \leq l$ in general. 
Remark 13. If $g=1$ in Proposition 12 , then $\cup^{i} \mathcal{G}=\Delta^{i} \mathcal{G}$ for any $i$, and the case is covered in Theorem 9 (b).

Proposition 14. Let $\mathcal{G}=\cup_{i=1}^{l} \mathcal{G}_{i}$ be a family of sets where each $\mathcal{G}_{i}$ is union closed and $A \cap B=\emptyset$ for $A \in \mathcal{G}_{i}$ and $B \in \mathcal{G}_{j}(i \neq j)$. Assume there is an $i_{0}$ and an $x \in \mathcal{G}_{i_{0}}$ such that $x$ is in at least $\left(\left|\mathcal{G}_{i_{0}}\right|+1\right) / 2$ of the sets of $\mathcal{G}_{i_{0}}$. Then the union-closed conjecture is true for $\cup^{l} \mathcal{G}$ and thus, $h_{\cup}(\mathcal{G}, x) \leq l$.

Proof. Let $\left|\mathcal{G}_{i}\right|=k_{i}$. Without loss of generality, let $x$ be in at least $\left(k_{1}+1\right) / 2$ sets of $\mathcal{G}_{1}$. We will show that $\left|\cup^{l}(\mathcal{G}, x)\right| \geq \frac{\left|\cup^{l} \mathcal{G}\right|}{2}$.

Form a complete $l$ partite graph $H$ whose parts are the $\mathcal{G}_{i}$ families $1 \leq$ $i \leq l$. Then $\left|\cup^{l} \mathcal{G}\right|$ is the total number of cliques in $H$ and $\left|\cup^{l}(\mathcal{G}, x)\right|$ is the total number of cliques containing a vertex $A \in \cup_{i=1}^{l} \mathcal{G}_{i}$ such that $x \in A$. So

$$
\begin{aligned}
\left|\cup^{l} \mathcal{G}\right|= & \sum_{i=1}^{l} k_{i}+\sum_{1 \leq i_{1}<i_{2} \leq l} k_{i_{1}} k_{i_{2}}+\cdots \\
& +\sum_{1 \leq i_{1}<\cdots<i_{l-1} \leq l} k_{i_{1}} k_{i_{2}} \cdots k_{i_{l-1}}+k_{1} k_{2} \cdots k_{l}
\end{aligned}
$$

and

$$
\begin{aligned}
\left|\cup^{l}(\mathcal{G}, x)\right|= & \frac{1+k_{1}}{2}+\frac{1+k_{1}}{2} \sum_{i=2}^{l} k_{i}+\frac{1+k_{1}}{2} \sum_{2 \leq i_{1}<i_{2} \leq l} k_{i_{1}} k_{i_{2}}+\cdots \\
& +\frac{1+k_{1}}{2} \sum_{2 \leq i_{1}<\cdots<i_{l-2} \leq l} k_{i_{1}} k_{i_{2}} \cdots k_{i_{l-2}}+\frac{1+k_{1}}{2} k_{2} \cdots k_{l} \\
(11) \quad & \frac{1}{2}+\frac{1}{2}\left(k_{1}+\sum_{i=2}^{l} k_{i}\right)+\frac{1}{2}\left(k_{1} \sum_{i=2}^{l} k_{i}+\sum_{2 \leq i_{1}<i_{2} \leq l} k_{i_{1}} k_{i_{2}}\right)+\cdots \\
& +\frac{1}{2}\left(k_{1} \sum_{2 \leq i_{1}<\cdots<i_{l-2} \leq l} k_{i_{1}} k_{i_{2}} \cdots k_{i_{l-2}}+k_{2} \cdots k_{l-1}\right)+\frac{k_{1}}{2} k_{2} \cdots k_{l-1} .
\end{aligned}
$$

From (11) and the expression of $\left|\cup^{l} \mathcal{G}\right|$ above, we obtain

$$
\begin{aligned}
\left|\cup^{l}(\mathcal{G}, x)\right|= & \frac{1}{2}+\frac{1}{2} \sum_{i=1}^{l} k_{i}+\frac{1}{2} \sum_{1 \leq i_{1}<i_{2} \leq l} k_{i_{1}} k_{i_{2}}+\cdots \\
& +\frac{1}{2} \sum_{1 \leq i_{1}<\cdots<i_{l-1} \leq l} k_{i_{1}} k_{i_{2}} \cdots k_{i_{l-1}}+\frac{1}{2} k_{1} k_{2} \cdots k_{l} \\
> & \frac{\left|\cup^{l} \mathcal{G}\right|}{2} .
\end{aligned}
$$




\section{Some special cases of union-closed conjecture}

Let $\mathcal{G}=\left\{A_{1}, \ldots, A_{l}\right\}$ be a family of $l$ sets. We follow the same notations as in the previous section. Note that $\cup^{l} \mathcal{G}$ is the union-closed family of sets generated by the sets in $\mathcal{G}$.

Definition 15. We say that $\left\{\left\{A_{i_{1}}, \ldots, A_{i_{s}}\right\},\left\{A_{j_{1}}, \ldots, A_{j_{t}}\right\}\right\}$ is an overcount in $\cup^{n} \mathcal{G}$ (resp. $\cup^{n}(\mathcal{G}, x)$ ) if the following conditions hold

(i) $A_{i_{1}} \cup \cdots \cup A_{i_{s}}$ and $A_{j_{1}} \cup \cdots \cup A_{j_{t}}$ are in $\cup^{n} \mathcal{G}$ (resp. $\cup^{n}(\mathcal{G}, x)$ ),

(ii) $\left\{A_{i_{1}}, \ldots, A_{i_{s}}\right\} \neq\left\{A_{j_{1}}, \ldots, A_{j_{t}}\right\}$,

(iii) $A_{i_{1}} \cup \cdots \cup A_{i_{s}}=A_{j_{1}} \cup \cdots \cup A_{j_{t}}$.

We define an auxiliary graph $H^{n}=\left(V, E^{n}\right)\left(\right.$ resp. $\left.H_{x}^{n}=\left(V, E_{x}^{n}\right)\right)$ corresponding to the overcounts in $\cup^{n} \mathcal{G}\left(\operatorname{resp} . \cup^{n}(\mathcal{G}, x)\right)$ as follows. Let

$$
V=\left\{\left\{A_{i_{1}}, \ldots, A_{i_{s}}\right\}: 1 \leq i_{1}<\cdots<i_{s} \leq n \leq l\right\}
$$

and join $L \in V$ and $R \in V$ by an edge in $H^{n}$ (resp. $H_{x}^{n}$ ) if $\{L, R\}$ is an overcount in $\cup^{n} \mathcal{G}\left(\right.$ resp. $\left.\cup^{n}(\mathcal{G}, x)\right)$.

Definition 16. A set $\mathcal{O}$ of overcounts in $\cup^{n} \mathcal{G}$ (or $\cup^{n}(\mathcal{G}, x)$ ) is independent if the corresponding edges in graph $H^{n}$ (resp. $\left.H_{x}^{n}\right)$ do not induce a cycle.

Lemma 17. Let $\mathcal{G}=\left\{A_{1}, \ldots, A_{l}\right\}$ be a family of $l$ sets. Let $x \in \mathcal{G}_{\mathrm{gd}}$ and $c_{x}$ be the maximum number of independent overcounts in $\cup^{n}(\mathcal{G}, x)$. Let $c$ be the maximum number of independent overcounts in $\cup^{n} \mathcal{G}$. Suppose that $x$ is in $q$ sets $A_{i} \in \mathcal{G}$. Then, $2\left|\cup^{n}(\mathcal{G}, x)\right|-\left|\cup^{n} \mathcal{G}\right| \geq 0$ if and only if

$$
c-2 c_{x} \geq 2 \sum_{i=1}^{n}\left(\begin{array}{c}
l-q \\
i
\end{array}\right)-\sum_{i=1}^{n}\left(\begin{array}{l}
l \\
i
\end{array}\right) .
$$

Proof. Without loss of generality, we assume that $x \in A_{i}$ for $1 \leq i \leq q$ and $x \notin A_{i}$ for $i>q$. Let us estimate $\left|\cup^{n} \mathcal{G}\right|$ and $\left|\cup^{n}(\mathcal{G}, x)\right|$.

Note that every set in $\cup^{n} \mathcal{G}$ is of the form $A_{i_{1}} \cup \cdots \cup A_{i_{s}}$, where $1 \leq s \leq n$. If $A_{i_{1}} \cup \cdots \cup A_{i_{s}}$ are pairwise distinct, then $\left|\cup^{n} \mathcal{G}\right|=\sum_{i=1}^{n}\left(\begin{array}{l}l \\ i\end{array}\right)$. In general,

$$
\left|\cup^{n} \mathcal{G}\right|=\sum_{i=1}^{n}\left(\begin{array}{l}
l \\
i
\end{array}\right)-c
$$

Similarly, every set in $\cup^{n}(\mathcal{G}, x)$ is of the form $A_{i_{1}} \cup \cdots \cup A_{i_{s}}$, where $1 \leq s \leq n$ and $1 \leq i_{1} \leq q$. If all these $A_{i_{1}} \cup \cdots \cup A_{i_{s}}$ are distinct, then $\left|\cup^{n}(\mathcal{G}, x)\right|=$ 
$\sum_{i=1}^{n}\left(\begin{array}{l}l \\ i\end{array}\right)-\sum_{i=1}^{n}\left(\begin{array}{c}l-q \\ i\end{array}\right)$. In general

$$
\left|\cup^{n}(\mathcal{G}, x)\right|=\sum_{i=1}^{n}\left(\begin{array}{l}
l \\
i
\end{array}\right)-\sum_{i=1}^{n}\left(\begin{array}{c}
l-q \\
i
\end{array}\right)-c_{x} .
$$

By (12) and (13), we see that $2\left|\cup^{n}(\mathcal{G}, x)\right|-\left|\cup^{n} \mathcal{G}\right| \geq 0$ is equivalent to $c-2 c_{x} \geq 2 \sum_{i=1}^{n}\left(\begin{array}{c}l-q \\ i\end{array}\right)-\sum_{i=1}^{n}\left(\begin{array}{l}l \\ i\end{array}\right)$.

By taking $l=n$ in the above lemma, we obtain the following results confirming some special cases of the union-closed conjecture.

Theorem 18. Let $\mathcal{G}=\left\{A_{1}, \ldots, A_{l}\right\}$ be a family of $l$ sets. Let $x \in \cup_{i=1}^{l} A_{i}$ and $c_{x}$ be the maximum number of independent overcounts in $\cup^{l}(\mathcal{G}, x)$. Let $c$ be the maximum number of independent overcounts in $\cup^{l} \mathcal{G}$ and $\gamma=c-c_{x}$. Suppose that $x$ is in $q$ sets $A_{i} \in \mathcal{G}$.

(1) The union-closed conjecture is true for $\cup^{l} \mathcal{G}$ if and only if there exists an $x \in \cup_{i=1}^{l} A_{i}$ such that $c \geq 2 c_{x}-2^{l}+2^{l-q+1}-1$.

(2) In particular, the union-closed conjecture is true for $\cup^{l} \mathcal{G}$ if one of the following conditions holds:

(2.a) $c_{x} \leq 2^{l}-2^{l-q+1}+1$.

(2.b) $\gamma \geq 2^{l-q}-1-q$.

(2.c) $2^{l-q} \leq q+1$.

(2.d) $\left|\cup^{l} \mathcal{G}\right| \geq 2^{l-q+1}-2$.

Proof. Taking $n=l$ in (12) and (13), we have

$$
\left|\cup^{l} \mathcal{G}\right|=2^{l}-1-c \text { and }\left|\cup^{l}(\mathcal{G}, x)\right|=2^{l}-2^{l-q}-c_{x} .
$$

(1) By (14), we see that $2\left|\cup^{l}(\mathcal{G}, x)\right|-\left|\cup^{l} \mathcal{G}\right| \geq 0$ is equivalent to $c \geq$ $2 c_{x}-2^{l}+2^{l-q+1}-1$.

(2.a) Note that $c \geq c_{x}$. If $c_{x} \leq 2^{l}-2^{l-q+1}+1$, then (14) yield

$$
2\left|\cup^{l}(\mathcal{G}, x)\right|-\left|\cup^{l} \mathcal{G}\right| \geq 2^{l}-2^{l-q+1}+1-c_{x} \geq 0 .
$$

(2.b) Note that $\left|\cup^{l}(\mathcal{G}, x)\right| \geq q$, so $c_{x} \leq 2^{l}-2^{l-q}-q$. If $\gamma=c-c_{x} \geq 2^{l-q}-1-q$, then (14) yield

$$
2\left|\cup^{l}(\mathcal{G}, x)\right|-\left|\cup^{l} \mathcal{G}\right| \geq 2^{l}-2^{l-q+1}+1-c_{x}+\gamma \geq 0,
$$

because $c_{x} \leq 2^{l}-2^{l-q}-q$ and $\gamma \geq 2^{l-q}-1-q$. 
(2.c) Since $c_{x} \leq 2^{l}-2^{l-q}-q$ and $2^{l-q} \leq q+1$, then equations (14) yield

$$
2\left|\cup^{l}(\mathcal{G}, x)\right|-\left|\cup^{l} \mathcal{G}\right| \geq 2^{l}-2^{l-q+1}+1-c_{x} \geq 0 .
$$

(2.d) If $\left|\cup^{l} \mathcal{G}\right| \geq 2^{l-q+1}-2$, then equation (14) yields $c \leq 2^{l}-2^{l-q+1}+1$. Since $c_{x} \leq c$, then $c_{x} \leq 2^{l}-2^{l-q+1}+1$ and (2.d) follows from (2.a).

The next corollary follows directly from Theorem 18 (2.d) and the fact that the union-closed conjecture holds for the families with a generating family of pairwise disjoint sets.

Corollary 19. The union-closed conjecture holds for a union-closed family $\mathcal{F}$ of sets if $\mathcal{F}$ has a generating family of sets $\mathcal{G}$ with $|\mathcal{G}| \leq \log _{2}(|\mathcal{F}|+2)+1$.

Given a family of sets $\mathcal{G}$ with $l$ sets, we say that $\cup^{l} \mathcal{G}$ satisfies the averaged Fránkl's property if

$$
\sum_{x \in \mathcal{G}_{g d}}\left(2\left|\cup^{l}(\mathcal{G}, x)\right|-\left|\cup^{l} \mathcal{G}\right|\right) \geq 0
$$

Satisfying the averaged Fránkl's property clearly implies satisfying the unionclosed conjecture. As observed in [2], there are many families $\mathcal{G}$ with $l$ sets such that $\cup^{l} \mathcal{G}$ satisfying the union-closed conjecture, but the averaged Fránkl's property fails.

For any family of sets $\mathcal{G}=\left\{A_{1}, \ldots, A_{l}\right\}$, recall that the ground set of $\mathcal{G}$ is $\mathcal{G}_{\mathrm{gd}}=\cup_{i=1}^{l} A_{i}$. For any $x \in \mathcal{G}_{\mathrm{gd}}$, we let

$$
q_{x}(\mathcal{G})=\{A: x \in A \in \mathcal{G}\} \text { and } q_{\min }(\mathcal{G})=\min _{x \in \mathcal{G}_{\mathrm{gd}}} q_{x}(\mathcal{G}) .
$$

Furthermore, we sometimes write $q_{x}$ (resp. $q_{\text {min }}$ ) instead of $q_{x}(\mathcal{G})$ (resp. $\left.q_{\min }(\mathcal{G})\right)$ if the family $\mathcal{G}$ is clear from the context.

Let $\mathcal{O}$ be a maximum independent set of overcounts in $\cup^{l} \mathcal{G}$. Then for any overcount $W=\{L, R\} \in \mathcal{O}$, we let $S_{W}=\bigcup_{A \in L} A=\bigcup_{A \in R} A$. Then $S_{W}$ is a union of some sets $A_{i} \in \mathcal{G}$. Define the average size of a set $S_{W}$ over all $W \in \mathcal{O}$ by

$$
\bar{s}(\mathcal{G})=\frac{1}{|\mathcal{O}|} \sum_{W \in \mathcal{O}}\left|S_{W}\right| .
$$

Let $c_{x}$ be the maximum number of independent overcounts in $\cup^{l}(\mathcal{G}, x)$ and define the average value of $c_{x}$ over all $x \in \mathcal{G}_{\mathrm{gd}}$ by

$$
\bar{c}(\mathcal{G})=\frac{1}{\left|\mathcal{G}_{\mathrm{gd}}\right|} \sum_{x \in \mathcal{G}_{\mathrm{gd}}} c_{x} .
$$


Theorem 20. Let $\mathcal{G}$ be a family of $l$ sets with $g=\left|\mathcal{G}_{\text {gd }}\right|$. Let $\bar{s}=\bar{s}(\mathcal{G})$ and $\bar{c}=\bar{c}(\mathcal{G})$ be as defined in (15) and (16) respectively. Then $g / \bar{s} \geq 1$ always holds.

(i) The averaged Fránkl's property is true for $\cup^{l} \mathcal{G}$ if and only if

$$
(2-g / \bar{s}) \bar{c} \leq 1+2^{l}-\left(2^{l} / g\right) \sum_{x \in \mathcal{G} g d} \frac{1}{2^{q_{x}-1}} .
$$

In particular, the averaged Fránkl's property is true for $\cup^{l} \mathcal{G}$ if $g / \bar{s} \geq 2$.

(ii) The union-closed conjecture is true for $\cup^{l} \mathcal{G}$ if $1 \leq(g / \bar{s})<2$, and there exists $x \in \mathcal{G}_{\text {gd }}$ satisfying

$$
c_{x} \leq \min \left\{\frac{2^{l}-2^{l-q_{x}+1}+1}{2-g / \bar{s}}, \bar{c}\right\} .
$$

In particular, the union-closed conjecture is true for $\cup^{l} \mathcal{G}$ if $\bar{c} \leq \frac{2^{l}-2^{l-q_{x}+1}+1}{2-g / \bar{s}}$. (iii) The union-closed conjecture is true for $\cup^{l} \mathcal{G}$ for any positive number $\epsilon<1$ with

$$
1+\epsilon \leq g / \bar{s}<2 \text { and } q_{\min } \geq 1-\log _{2}(\epsilon) .
$$

Moreover, by combining $(i)$ and $($ iii $)$, it follows that the union-closed conjecture is true for $\cup^{l} \mathcal{G}$ whenever $g / \bar{s}>1$ and $q_{\min } \geq 1-\log _{2}(g / \bar{s}-1)$.

Proof. Let $\mathcal{O}$ be a maximum independent set of overcounts in $\cup^{l} \mathcal{G}$. For any $x \in \mathcal{G}_{\text {gd }}$, let $\mathcal{O}_{x} \subseteq \mathcal{O}$ denote the (possibly empty) set of all those overcounts $W=\{L, R\} \in \mathcal{O}$ for which $x \in S_{W}=\bigcup_{A \in L} A=\bigcup_{A \in R} A$. We count in two ways the number of pairs $(x, W)$ such that $x \in \mathcal{G}_{\text {gd }}$ and $W \in \mathcal{O}_{x}$. Then we have

$$
\sum_{W \in \mathcal{O}}\left|S_{W}\right|=\sum_{x \in \mathcal{G}_{\text {gd }}}\left|\mathcal{O}_{x}\right|
$$

Let $c=|\mathcal{O}|$, and let $C_{x}$ be a maximum independent set of overcounts in $\cup^{l}(\mathcal{G}, x)$ with $c_{x}=\left|C_{x}\right|$. If $\left|C_{x}\right|>\left|\mathcal{O}_{x}\right|$ then $\mathcal{O}^{\prime}=\left(\mathcal{O} \backslash \mathcal{O}_{x}\right) \cup C_{x}$ is also an independent set of overcounts in $\cup^{l} \mathcal{G}$ with $\left|\mathcal{O}^{\prime}\right|>|\mathcal{O}|$, which contradicts $\mathcal{O}$ being of maximum size. So we may assume that $c_{x}=\left|C_{x}\right|=\left|\mathcal{O}_{x}\right|$ for any $x \in \mathcal{G}_{\text {gd }}$. Then (17) yields

$$
\sum_{W \in \mathcal{O}}\left|S_{W}\right|=\sum_{x \in \mathcal{G}_{\text {gd }}} c_{x}
$$


Now, it follows from (18) and the definitions of $\bar{s}$ and $\bar{c}$ (see (15) and (16)) that

$$
c=(g / \bar{s}) \cdot \bar{c} .
$$

In general $g \geq \bar{s}$ since $S_{W} \subseteq \mathcal{G}_{\text {gd }}$ for all $W \in \mathcal{O}$.

(i) Applying (14), we have $\sum_{x \in \mathcal{G}_{g d}}\left(2\left|\cup^{l}(\mathcal{G}, x)\right|-\left|\cup^{l} \mathcal{G}\right|\right) \geq 0$ if and only if

$$
\sum_{x \in \mathcal{G}_{g d}}\left(2\left|\cup^{l}(\mathcal{G}, x)\right|-\left|\cup^{l} \mathcal{G}\right|\right)=\sum_{x \in \mathcal{G}_{g d}}\left(2\left(2^{l}-2^{l-q_{x}}-c_{x}\right)-\left(2^{l}-1-c\right)\right) \geq 0
$$

if and only if

$$
c \geq \frac{2 \sum_{x \in \mathcal{G}_{\mathrm{gd}}} c_{x}}{g}+\frac{2^{l}}{g} \sum_{x \in \mathcal{G}_{\mathrm{gd}}} \frac{1}{2^{q_{x}-1}}-2^{l}-1,
$$

if and only if

$$
c \geq 2 \bar{c}+\frac{2^{l}}{g} \sum_{x \in \mathcal{G}_{\mathrm{gd}}} \frac{1}{2^{q_{x}-1}}-2^{l}-1 .
$$

By (19), the above inequality holds if and only if

$$
(2-g / \bar{s}) \bar{c} \leq 1+2^{l}-\frac{2^{l}}{g} \sum_{x \in \mathcal{G} g \mathrm{~d}} \frac{1}{2^{q_{x}-1}} .
$$

If there exists $x \in \mathcal{G}_{\text {gd }}$ satisfying

$$
c_{x} \leq \min \left\{\frac{2^{l}-2^{l-q_{x}+1}+1}{2-g / \bar{s}}, \bar{c}\right\},
$$

then by (19), we obtain

$$
c=(g / \bar{s}) \cdot \bar{c} \geq(g / \bar{s}) c_{x} \geq 2 c_{x}-2^{l}+2^{l-q_{x}+1}-1,
$$

because $c_{x} \leq\left(2^{l}-2^{l-q_{x}+1}+1\right) /(2-g / \bar{s})$ holds by hypothesis. Now (ii) follows from Theorem 18 (2.b).

To prove (iii), first note $2-g / \bar{s} \leq 1-\epsilon$ since (by hypothesis) $g / \bar{s} \geq 1+\epsilon$. Consequently, the sufficient condition in (ii), namely $\bar{c} \leq\left(2^{l}-2^{l-q_{x}+1}+\right.$ 1) $/(2-g / \bar{s})$, holds if

$$
\bar{c} \leq(1-\epsilon)^{-1} \cdot\left(2^{l}-2^{l-q_{x}+1}+1\right) .
$$


Now (20) holds because

$$
\bar{c} \leq 2^{l} \leq(1-\epsilon)^{-1} \cdot\left(2^{l}-2^{l-q_{x}+1}+1\right),
$$

where the last inequality holds since $\epsilon<1$ and

$$
q_{x} \geq q_{\min } \geq 1-\log _{2}(\epsilon) \Rightarrow 2^{l} \leq(1-\epsilon)^{-1} \cdot\left(2^{l}-2^{l-q_{x}+1}+1\right) .
$$

The proof of part (iii) is now complete.

The last statement of the theorem is a straightforward combination of (i) and (iii).

\section{References}

[1] I. Bosnak and P. Markovic, The 11-element case of Fránkl's conjecture. Electr. J. Combin., 15 (2008), \#R88.

[2] G. Czédli, On average Fránkl's conjecture for large union-closed-sets. J. Combin. Theory Ser. A, 116 (2009), no. 3, 724-729. MR2500167

[3] K. Dohmen, A new perspective on the union-closed sets conjecture. Ars Combin., 58 (2001), 183-185. MR1820199

[4] P. Fránkl, Extremal Set Systems. Chapter 24 in Handbook of Combinatorics (MIT Press \& North-Holland, 1995), p. 1296. MR1373680

[5] W. Gao and H. Yu, Note on the union-closed sets conjecture. Ars Combin., 49 (1998), 280-288. MR1633064

[6] R. T. Johnson and T. P. Vaughan, On union-closed families. I. J. Combin. Theory Ser. A, 84 (1998), 242-249, and J. Combin. Theory Ser. A, 85 (1999), 112-119. MR1659436

[7] G. Lo Faro, A note on the union-closed sets conjecture. J. Austral. Math. Soc. Ser. A, 57 (1994), 230-236. MR1288674

[8] R. M. Norton and D. G. Sarvate, A note of the union-closed sets conjecture. J. Austral. Math. Soc. Ser. A, 55 (1993), 411-413. MR1243780

[9] B. Poonen, Union-closed families. J. Combin. Theory Ser. A, 59 (1992), 253-268. MR1149898

[10] I. T. Roberts and J. Simpson, A note on the union-closed sets conjecture. Australas. J. Combin., 47 (2010), 265-267. MR2662546

[11] D. G. Sarvate and J. C. Renaud, On the union-closed sets conjecture. Ars Combin., 27 (1989), 149-153. MR0989460 
[12] D. G. Sarvate and J. C. Renaud, Improved bounds for the union-closed sets conjecture. Ars Combin., 29 (1990), 181-185. MR1046106

YUeJiAn PEng

SChoOl OF Mathematics

HUNAN UNIVERSITY

Changsha 410082

P.R. CHINA

E-mail address: ypeng1@163.com

PAPA SisSOKHO

Mathematics Department

ILLINOIS STATE UNIVERSITY

NORMAL, ILLINOIS 61790-4520

USA

E-mail address: psissok@ilstu.edu

Cheng Zhao

Department of Mathematics and Computer Science

INDiana State University

Terre Haute, Indiana 47809

USA

AND

SChool of Mathematics

JiLin University, ChangChun

P.R. CHINA

E-mail address: cheng.zhao@indstate.edu

Received December 282011 